\title{
Network Architectures Exploiting Multiple Tethered Balloon Constellations for Coverage Extension
}

\author{
S. H. Alsamhi ${ }^{1}$, Sachin Kumar Gapta ${ }^{2}$, N. S. Rajput ${ }^{3}$, and R. K. Saket ${ }^{4}$
}

\begin{abstract}
Base Stations (BS) is increasing drastically to cover large area and that leads to increase the cost. Therefore, instead of increasing the number of BS, height of BS is required to be increased. In this paper, we propose to use Tethered Balloon for carrying BS to altitude 200-440 meter. Therefore, it is able to provide effective and efficient telecommunication facilities and service with low cost. Additionally, the multi-Tethered Balloons concept has been used to extend the coverage area especially in thinly populated and remote areas. This paper describes possible multiple Tethered Balloons constellations, and explains how it is possible to increase the capacity. In this case, the WiMAX station is allocated in Tethered Balloon payload. Then, Quality of Service (QoS) parameters are measured and simulated for multiple Tethered Balloons using Optimized Network Engineering Tool (OPNET) Modeler 14.5 for the network implementation. The performance of our proposed technology is effectively and efficiently for delivering broadband communication services for large coverage areas.
\end{abstract}

Keywords-Tethered Balloon, Network Architecture of Multi Tethered Balloon, OPNET, WiMAX.

\section{INTRODUCTION}

$\mathrm{G}^{\mathrm{s}}$ ROWING demand for wireless communication seeks higher data rates and reconfigurable support. Various developments in the communication technologies and applications such as voice, internet access, sensing, video, monitoring, navigation etc. made people look for better services in low cost. Furthermore, for providing large coverage area, many towers and operational expenditure is required. Due to continuous demand to deliver higher capacity services, challenges for the terrestrial system have also increased because of limited radio spectrum resource. When the number of towers increase, it would result to increase the level of public exposure to microwave energy over time as well as cost will be increased especially in a rural coverage area. $^{1}$

S. H. Alsamhi is with the Department of Electronics Engineering, IBB University, Ibb, Yemen and Indian Institute of Technology (BHU), Varanasi221005, (Uttar Pradesh), India (e-mail: s.alsamhi.rs.ece@iitbhu.ac.in).

Sachin Kumar Gupta is with the Department of Electronics and Communication Engineering, SMVD University, Katra-182320 (Jammu \& Kashmir) and Indian Institute of Technology (BHU), Varanasi-221005, (Uttar Pradesh,) India (e-mail: sachin.rs.eee@ iitbhu.ac.in).

N. S. Rajput is with the Department of Electronics, Indian Institute of Technology (BHU), Varanasi-221005, (Uttar Pradesh), India (e-mail: nsrajput.ece@iitbhu.ac.in).

R. K. Saket is an Associate Professor with the Department of Electrical Engineering, Indian Institute of Technology (BHU), Varanasi-221005, (Uttar Pradesh,) India (e-mail: $\underline{\text { kssaket.eee @iitbhu.ac.in) }}$
Recently, competitive system has attracted the attention, offering solutions for the bottlenecks with the satellite system such as aerial platforms. Aerial platforms are capable of providing wireless communication and remote sensing. Their most important features are: lower cost of installation and maintenance, low delay etc. $[1,2]$. Aerial platform categories represented as High Altitude Platform (HAP) which placed in stratosphere layer and operated at an altitude of 17-22 Km [2]. Also, Medium Altitude Platform (MAP) and Low Altitude Platform (LAP) which placed in troposphere layer $[1,2]$ as shown in Fig. 1.

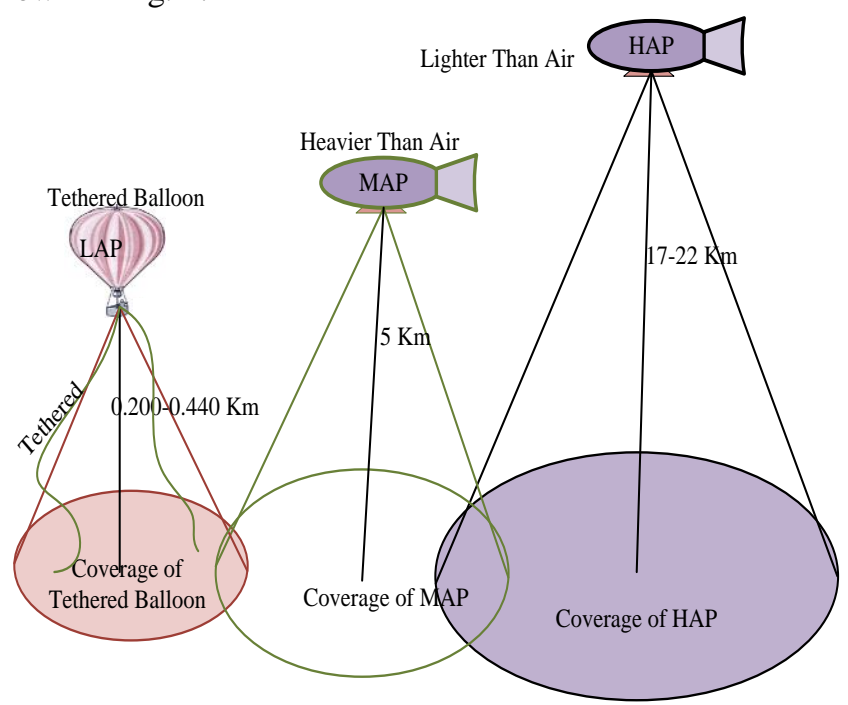

Fig. 1 Categories of aerial platform

Tethered Balloon is one of LAP families which operate at altitude 200- $440 \mathrm{~m}$ [2]. It is an inflated fabric structure, which classified as lighter than air (LTA). Also, it is restrained by a cable called 'tether' to maintain the balloon stable in its position. These tethers can also provide power for the payload from the ground [3]. Tethered Balloon has the potential to deliver wireless broadband service during the disastrous situation [4]. It also represents a vital solution for reducing environmental hazard through energy efficient of radio equipment and solar system.

Employing more than one Tethered Balloon to serve a common coverage area can significantly increase the capacity, 
i.e. number of supportable subscribers with Quality-of-Service (QoS). In this paper, using newly developed analysis and restriction mechanism for our proposed technology for extended the coverage and measured the performance of delivering broadband services.

The rest of this paper is organized as follows. In section II, Tethered Balloon technology is described. Section III provides coverage of Tethered Balloon. Section IV comes with the performance of delivering services. Network Scenario describes in section V. Result and discussions are given in section VI. Finally, conclusions are given in section VII.

\section{Tethered Balloon TeChNOLOGY}

Tethered Balloon is the one of the newest technology of wireless communication which operates at stratosphere layer for atmospheric research and subsequently and wireless communication. Zeppelin Company launched a craft in 2000, followed by many projects which focused on design of Tethered Balloon for various applications and accurate operation in different altitudes [5]. Then, in 2003, CAPANINA project focused on providing broadband services [6]. Internet connectivity all over the world using balloon network has proposed by Google Project 'Loon'. In 2001, A. Gawale et al., aimed to develop an airship for various applications such as surveillance, disaster management and advertisement [7]. This balloon established a long distance wireless communication link to provide Internet connectivity in rural areas [8]. Telecommunication and Broadband services in remote and hilly areas has been attempted [9].

Hence, this technology has the potential to deliver wide variety of telecommunication services. It overcomes the various challenges faced in traditional cellular systems such as limited coverage area, high power consumption, lack of Line of Sight (LoS) etc. Furthermore, this technology also carries the potential of mitigate the health risk due to exposure to radiation and environmental effects that are linked with terrestrial mobile BS. The following are the most importance advantages of Tethered Balloon [10,11]:

A. Low cost of launch and operation.

B. Very rapid deployment and accessible for maintenance.

C. Large coverage area compared with terrestrial systems.

D. Low propagation delay compared with satellite.

E. Line-of-sight links.

F. Shortest link length of space communication systems.

G. Easily reconfigurable.

Tethered Balloon has used for different applications such as passenger commutation, military applications, metrological usage as well. Recently, it is being used as platforms for lifting-up variety of payload (camera, radio antennas, electrooptical sensors, radio-relay equipment and advertising) for various research and applications. Some of the key applications have been listed as below:

A. Data collection balloon, such as measure temperature, humidity, pressure, weather monitoring [12], remote sensing [13].

B. Power beaming to supply ground equipment's [14].
C. Surveillance radar provides radar coverage to monitor civilian activities.

D. Enables wireless telecommunication services over large coverage area as well as support telecommunications in disaster area and healthy recovery [15].

Tethered is the connection link between balloon and other networks. It is assumed to be of circular section, sufficiently rough to ensure turbulent wakes at limited wind speed of 30 $\mathrm{m} / \mathrm{s}$, giving a drag coefficient of 0.3 [3]. Tethered varies with altitude, because tethered is assumed to be supported by balloon as well as the tethered winch is fixed to the ground. The advantages of using tethered are:

A. Minimized the need for propulsion to keep balloon in high winds.

B. Send communication signals and video feed.

C. Used for supply payload power in case of not using solar power.

D. Provide low-pressure waveguides to convey millimeter wave power.

The tethered balloon is usually connected to the other network using fiber for high-speed data transfer. It also provides a connection between Remote Radio Unit (RRU) and Mobile Unit (MU) to transmit power and data.

\section{Coverage Of Tethered Balloon}

Tethered Balloon is able to cover large coverage area and replace large number of terrestrial towers. The coverage can be estimated by the radiation power transmitted from Balloon. It can be extended by using multi-Tethered Balloons, and depends on many parameters such as radiation power, height of antenna, environment and propagation path. Cell radius calculation depends on the chosen value of allowable path loss. It is calculated from the link budget. The value of allowable path loss is also substituted in Hata model $[1,16]$ with 'correction factor' of small city taken as:

$$
\left(1.1 \log _{10}\left(f_{c}\right)-0.7\right) h_{r e}-\left(1.56 \log _{10}\left(f_{c}\right)-0.8\right)
$$

The variable D in Hata model equation determines the cell radius of the coverage area.

$$
\begin{gathered}
\mathrm{P}_{\mathrm{l}}=69.55+26.16 \log _{10}\left(\mathrm{f}_{\mathrm{c}}\right)-13.82 \log _{10}\left(\mathrm{~h}_{\mathrm{te}}\right) \\
-\mathrm{a}\left(\mathrm{h}_{\mathrm{re}}\right)+(44.9 \\
\left.-6.55 \log _{10}\left(\mathrm{~h}_{\mathrm{te}} \mathrm{h}\right)\right) \log _{10} \mathrm{D}
\end{gathered}
$$

Where $f_{c}$ is frequency, $h_{r e}$ represent the hight of mobile antenna, $h_{\text {te }}$ is base station antenna height, $a\left(h_{r e}\right)$ represent the correction factor, $\mathrm{P}_{\mathrm{P}}$ is the path loss and $\mathrm{D}$ represent the cell rang in $\mathrm{Km}$.

Tethered Balloon network architecture represents as a local multipoint distribution system, which is basically a radio technology that provides broadband to many customers from a single or multiple Balloons in the space.

\section{A. $\quad$ Single Tethered Balloon Scenario}

A single Tethered Balloon is envisaged for providing a communication network services for special events such as disaster recovery, rapid broadband employment over highly populated citied as shown in Fig. 2. 


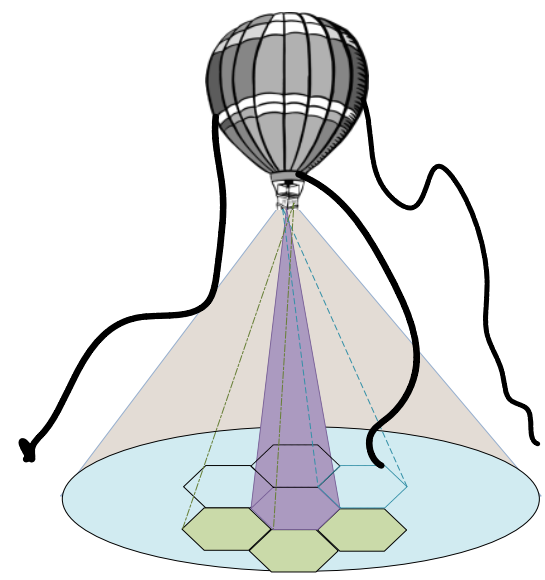

Fig. 2 Single tethered balloon

\section{B. Multi-Tethered Balloon}

The idea of increasing the number of Tethered Balloons serving a common coverage area is to increase the capacity and the bandwidth efficiency. Furthermore, Multi-Tethered Balloon constellations can increase the capacity by exploiting the directionality of the fixed user antenna. The reason for balloon configuration is to provide resilience and to provide unique diversity to end users receiving antennas for improved service availability. In the case of multi-Tethered Balloon constellations, they can be interconnected via ground stations or inter-balloon link (IBL) between two balloons represents an additional network interface. IBLs can be radio or optical, thus arbitrarily extending the system coverage. In fact, the coverage area is spilt into multiple cells to increasing the capacity. In addition, Multi-Tethered Balloon constellations could enhance the overall system capacity in overlapping coverage. A general Tethered Balloon constellations architecture is depicted in Fig. 3.

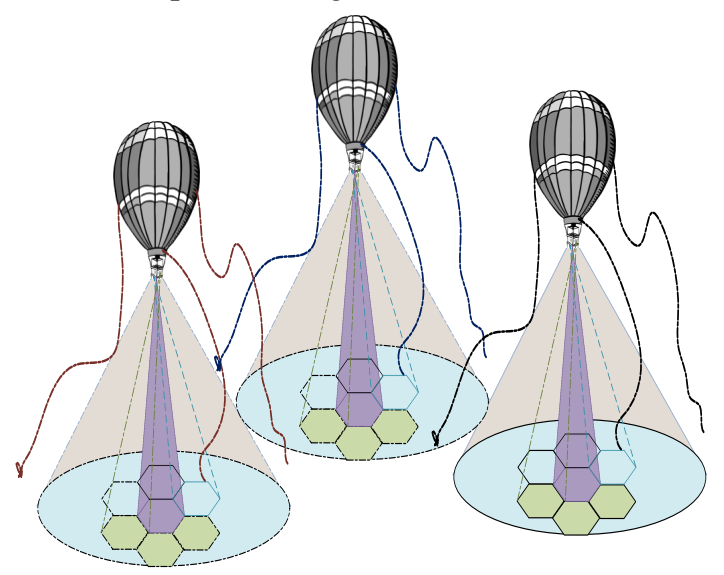

Fig. 3 Multiple tethered balloons

\section{Analysis The Delivery Services Performance}

The network performance evaluation needs different kinds of parameters. Generally, delay traffic and throughput parameters are used to test the network performance that is very important consideration in a communication network.

\section{A. Delay Analysis}

Delay specifies how long it takes for a bit of data to travel across the network from one user to another. It is typically measure in fractions of second. The packet end-to-end delay is the time of generation of a packet by the source up to the destination reception. This time is expressed in sec. Hence, all the delays in the network are called packet end-to-end delay. There are different kinds of activities because of which network delay is increased. We have several kinds of delays such as processing delay $D_{p}$, transmission delay $D_{t}$ and propagation delay $D_{d}$. Suppose, if there are N number of nodes then the total delay can be calculated by taking the average of all the packets, source destination pairs and network configuration.

$$
D_{e-e}=N\left(D_{p}+D_{t}+D_{d}\right)
$$

Where $D_{e-e}$ is end delay and $D_{t}$ is time it takes to push the packet's bit on link.

\section{B. Throughput Analysis}

Throughput is the number of data successfully delivered per unit time. It is controlled by available bandwidth and signal to noise ratio. Throughput is to be measure from the arrival of the first bit of data at the receiver. System throughput is the sum of the data rates that are delivered to all terminals in a network. System throughput is the fraction of time which channel is used for transmit payload bits successfully. It can be obtained by the probability events that may happened in randomly chosen slot time [2].

There are three random chosen slot correspondent's such as an idle slot, a collision, and a successful transmission which represented by probability of idle slot $\left(P_{\text {idel }}\right)$, probability of collision $\left(P_{c o l}\right)$ and probability of successful transmission $\left(P_{\text {suc }}\right)$ respectively. In addition, that probability occurred during particular time called idle slot time, collision time $T_{c o l}$ and successful transmission time $T_{\text {suc }}$, respectively. The average of duration time is represented by $T_{\text {avr }}$ and is given by (4).

$$
T_{\text {avr }}=P_{\text {idel }} \delta+P_{\text {succ }} T_{\text {succ }}+P_{\text {col }} T_{\text {col }} \ldots \ldots \ldots \ldots
$$

On the other hand, throughput is represented by $S$ and can be calculated by using (5).

$$
S=P_{\text {succ }} E[p] / T_{\text {avr }}
$$

Where, $E[p]$ is the payload information transmitting, then represented in (6).

$$
S=P_{\text {succ }} E[p] / P_{\text {idel }} \delta+P_{\text {succ }} T_{\text {succ }}+P_{\text {col }} T_{\text {col }}
$$

By dividing the numerator and denominator of equation (6) by $\left(P_{\text {succ }} T_{\text {succ }}\right)$, the throughput can be expressed as (7).

$$
\begin{aligned}
S=E[p] / T_{\text {suc }}[1 & +P_{\text {col }} / P_{\text {suc }}+T_{\text {col }} / T_{\text {suc }} \\
& \left.+P_{\text {idel }} / P_{\text {suc }}+\delta / T_{\text {suc }}\right]
\end{aligned}
$$

Accordingly, for computing the throughput for a given handshake, we only need to specify the corresponding values of $T_{c o l}$ and $T_{\text {suc }}$. 


\section{Design The Proposed Network}

Modeling and simulation of proposed network is using OPNET. OPNET is a real-time simulator designed mainly for the design and analysis of network models. The network topology used to carry out this research consists of two subnets are shown in Fig. 4. Each subnet consists of Tethered Balloon with WiMAX BS is allocated in the payload and numbers of subscriber are located in the coverage of each Tethered balloon as shown in Fig. 5. The purpose of this scenario was to examine the coverage and QoS deployment over Tethered Balloon network and to investigate the capability and deliver adequate QoS to voice applications.

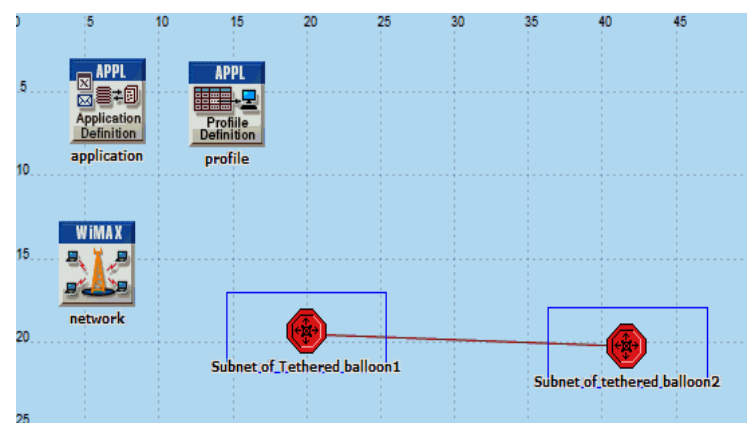

Fig. 4 Multiple tethered balloons

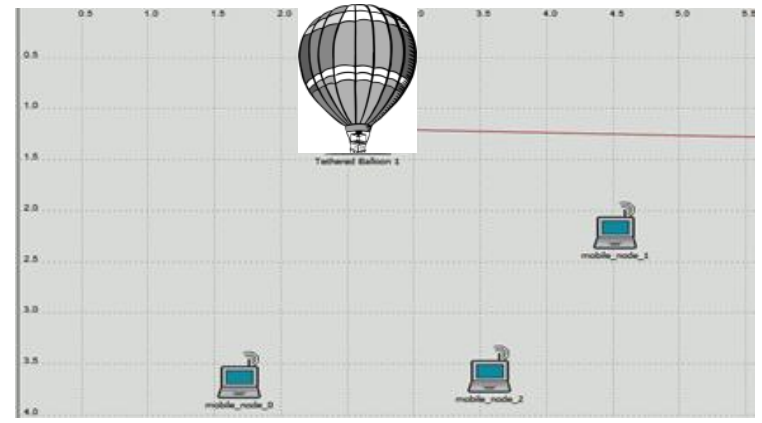

Fig. 5 Subnet of tethered balloon

\section{RESUlt AND Discussions}

This section contains the simulation results for scenario which are displayed for the proposed network using OPNET. For the analysis of results, some of QoS parameters are measured (i.e. delay, traffic and throughput for both scenarios).

Fig. 6 shows that delay increases from 0.0207 to 0.0204125 with increasing time significantly. Also the load and throughput are increased significantly. Load is increased from 33569.12 to 1151030 and throughput also increases from 33195.79 to 1151396.

Delay of different user is different that because of the different environments and different position from the center of Tethered Balloon as shown in Fig. 7. Tethered Balloon 2 delay is decreased from 0.224 to $0.222 \mathrm{Sec}$ as shown in Fig. 7. On the other hand, delay of mobile user nodes is different according to the position of user and the distance of user from the center coverage area of Tethered Balloon as shown in Fig. 7.

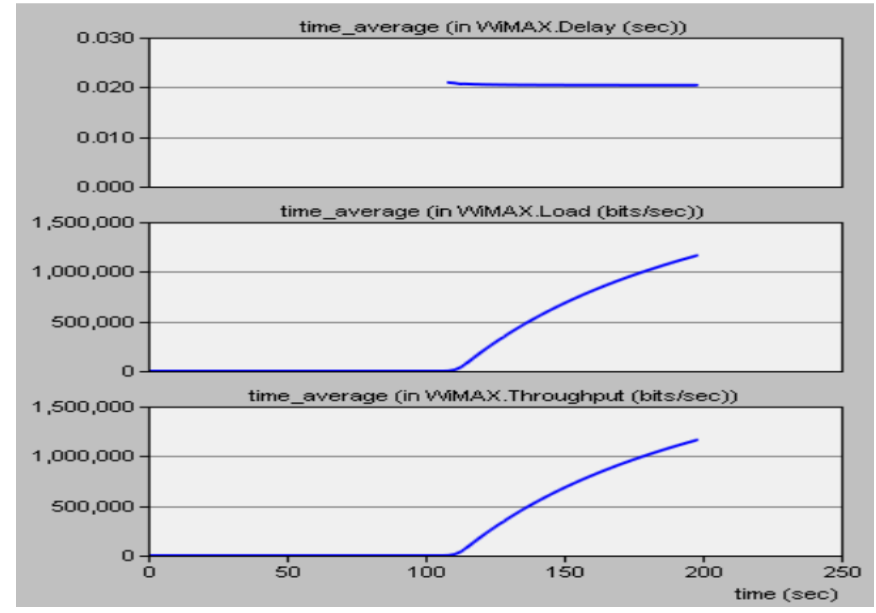

Fig. 6 Network global characteristics

Throughput in tethered balloon 2 is very high, but it is low in the mobile users. Throughput is increased with time as shown in Fig. 8. Traffic received and sent in case of subnet 0 is shown in Fig. 9.

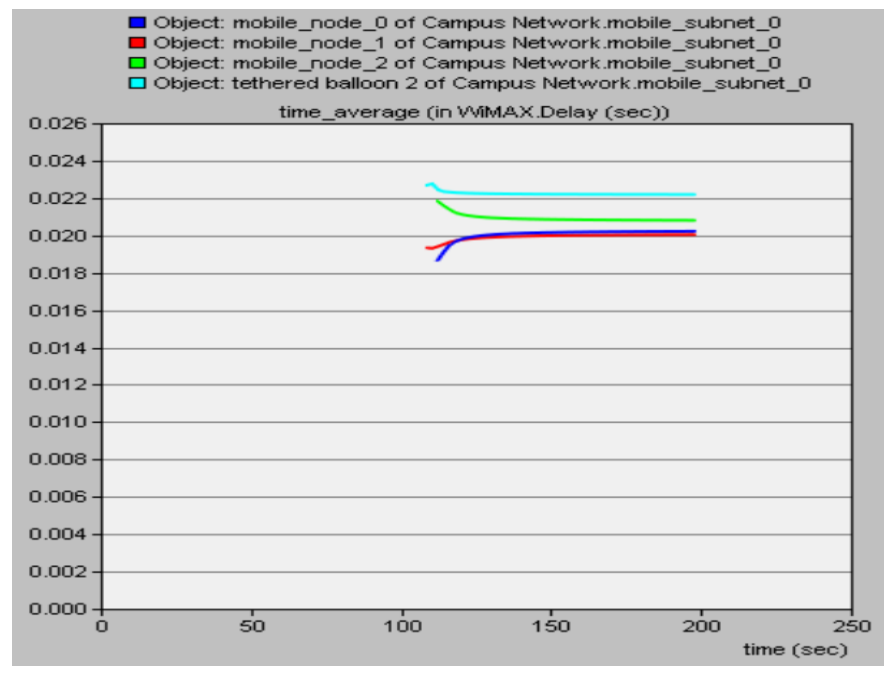

Fig. 7 Delay

Tethered Balloon 3 in subnet 1, delay is decreased from 0.0195 to $0.0181 \mathrm{Sec}$ as shown in Fig. 10. On the other hand, delay of mobile user nodes is different according to the position of user and the distance of user from the center coverage area of Tethered Balloon as shown in Fig. 10.

Throughput in tethered balloon 3 in subnet 1 is very high, but it is low in the mobile users. Throughput is increased with time as shown in Fig. 11. Traffic received and sent in case of subnet 1 is shown in Fig. 12. 


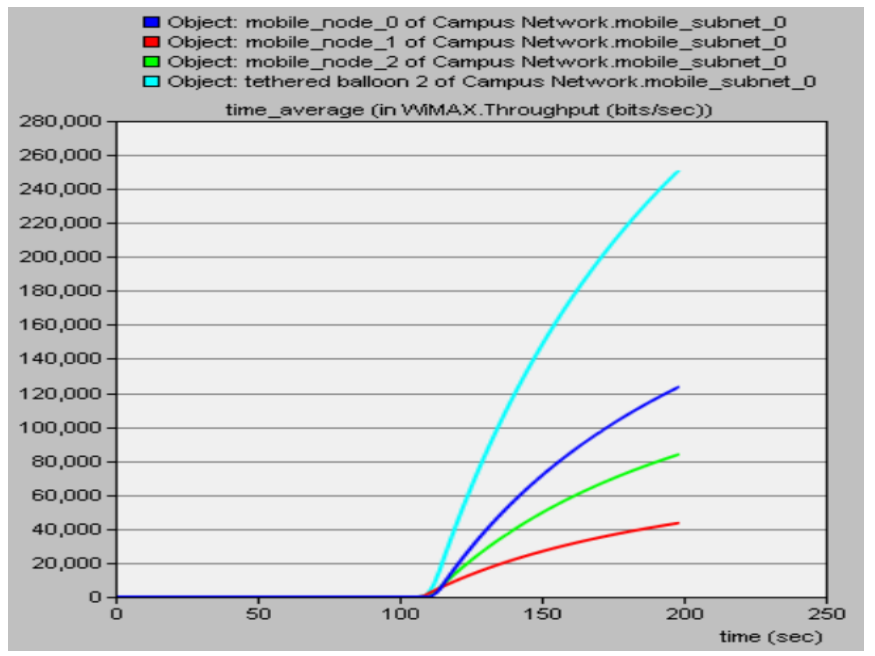

Fig. 8 Throughput of subnet 0

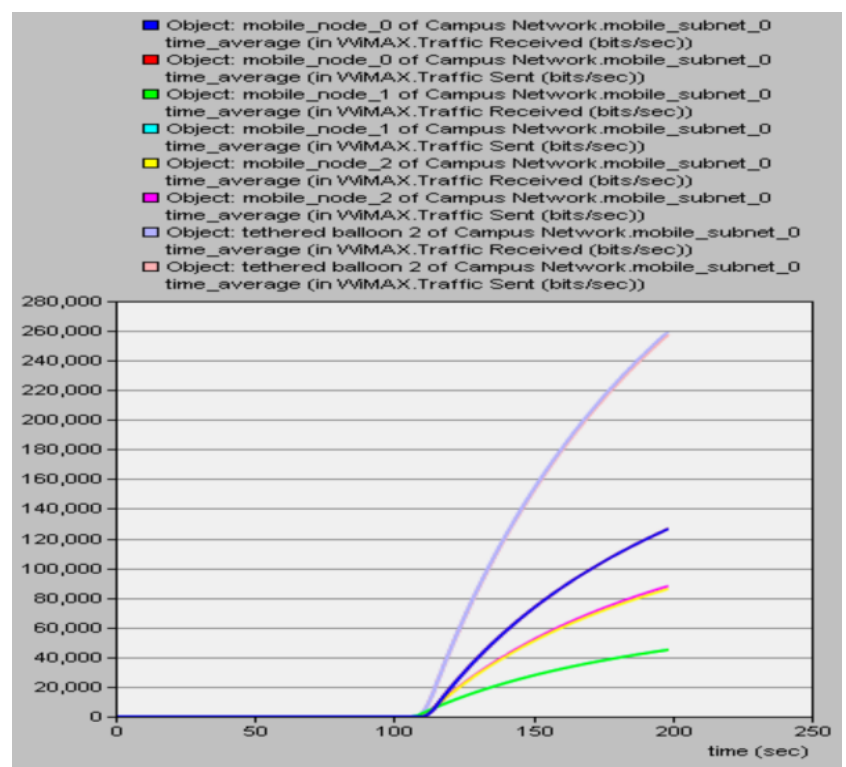

Fig. 9 Traffic of subnet 0

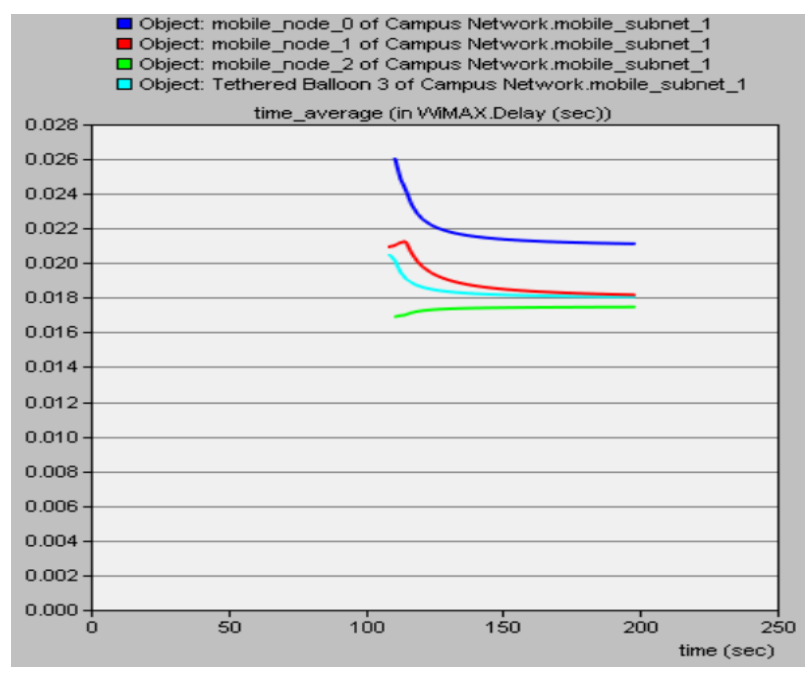

Fig. 10 Delay of subnet 1

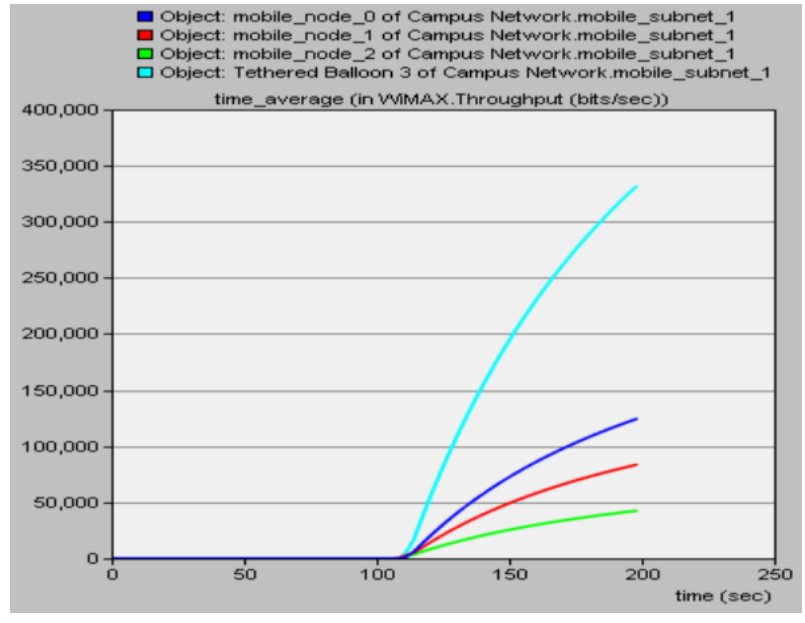

Fig. 11 Throughput of subnet 1

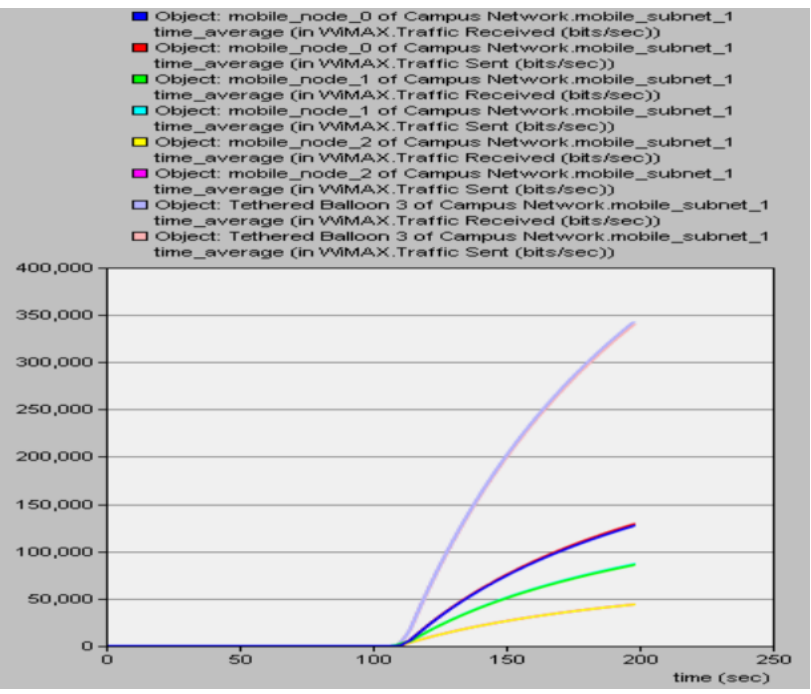

Fig. 12 Traffic of subnet 1

\section{CONCLUSION}

From simulation, we observe that the voice QoS in Tethered Balloon technology that carried WiMAX BS in its payload is influenced by several factors such as delay, throughput and traffic sent and received. Delay, throughput and traffic are the key parameters to measure the performance of QoS voice transmission in the Network. In this paper, we described how multi-Tethered Balloon constellations can be used for coverage extension. Different constellations of Tethered Balloons were applied in neighboring coverage areas. A performance analysis of a multi-Tethered Balloon in terms of QoS is presented. Results show that it is possible to improve the performance of QoS parameters in the coverage availability of Tethered Balloon.

\section{REFERENCES}

[1] S. Khaleefa, S. Alsamhi, and N. Rajput, "Tethered balloon technology for telecommunication, coverage and path loss," in Electrical, Electronics and Computer Science (SCEECS), 2014 IEEE Students' Conference on, 2014, pp. 1-4.

https://doi.org/10.1109/sceecs.2014.6804522 
[2] S. H. Alsamhi and N. Rajput, "An intelligent HAP for broadband wireless communications: Developments, QoS and applications," International Journal of Electronics and Electrical Engineering, vol. 3, 2015.

[3] N. Komerath, "An imaging, communications and beamed power architecture for first responders," in Proceedings of the 1st International Conference on Wireless Technologies for Humanitarian Relief, 2011, pp. 421-428.

https://doi.org/10.1145/2185216.2185326

[4] K. Mase, "How to deliver your message from/to a disaster area," IEEE Communications Magazine, vol. 49, pp. 52-57, 2011. https://doi.org/10.1109/MCOM.2011.5681015

[5] T. C. Tozer and D. Grace, "High-altitude platforms for wireless communications," Electronics \& Communication Engineering Journal, vol. 13, pp. 127-137, 2001. https://doi.org/10.1049/ecej:20010303

[6] D. Grace, M. Mohorcic, M. Oodo, M. Capstick, M. B. Pallavicini, and M. Lalovic, "CAPANINA-communications from aerial platform networks delivering broadband information for all," Proceedings of the 14th IST Mobile and Wireless and Communications Summit, 2005.

[7] A. Gawale, A. A. Raina, R. S. Pant, and Y. P. Jahagirdar, "Design fabrication and operation of low cost remotely controlled airships in india " in Proceedings of AIAA's 8th Aviation Technology Integration and Operations (ATIO) Conference, vol. 2008-8853, September 2008.

[8] P. Bilaye, V. Gawande, U. Desai, A. Raina, and R. Pant, "Low cost wireless internet access for rural areas using tethered aerostats," in Industrial and Information Systems, 2008. ICIIS 2008. IEEE Region 10 and the Third international Conference on, 2008, pp. 1-5. https://doi.org/10.1109/iciinfs.2008.4798405

[9] R. M. P. K. Chopra, R. Mehrotra, S. Jain;, "A New Topology for Telecom and Broad Band Services in Spars, Remote and Hilly Areas," WSEAS TRANSACTIONS on COMMUNICATIONS, vol. 10, pp. 273286, 2011.

[10] S. H. Alsamhi and N. S. Rajput, "An Intelligent HAP for Broadband Wireless Communications: Developments, QoS and Applications," International Journal of Electronics and Electrical Engineering, vol. 3, 2015.

[11] P. Bilaye, V. N. Gawande, U. B. Desai, A. A. Raina, and R. S. Pant, "Low Cost Wireless Internet Access for Rural Areas using Tethered Aerostats," in Industrial and Information Systems, 2008. ICIIS 2008. IEEE Region 10 and the Third international Conference on, 2008, pp. 15. https://doi.org/10.1109/iciinfs.2008.4798405

[12] P. Sankar and S. R. Norman, "Embedded system for monitoring atmospheric weather conditions using weather balloon," in Control, Automation, Communication and Energy Conservation, 2009. INCACEC 2009. 2009 International Conference on, 2009, pp. 1-4.

[13] M. Liu, G. Su, and Z. Lin, "The Application of Combined Camera in Low Altitude Remote Sensing With Unmanned Airship," in 2013 the International Conference on Remote Sensing, Environment and Transportation Engineering (RSETE 2013), 2013. https://doi.org/10.2991/rsete.2013.111

[14] R. Pant, N. Komerath, and A. Kar, "Application of lighter-than-air platforms for power beaming, generation and communications," in Electronic System Design (ISED), 2011 International Symposium on, 2011, pp. 242-247. https://doi.org/10.1109/ised.2011.51

[15] H. Hariyanto, H. Santoso, and A. K. Widiawan, "Emergency broadband access network using low altitude platform," in Instrumentation, Communications, Information Technology, and Biomedical Engineering (ICICI-BME), 2009 International Conference on, 2009, pp. 1-6. https://doi.org/10.1109/icici-bme.2009.5417275

[16] S. H. Alsamhi and N. S. Rajput, "Performance and Analysis of Propagation Models for Efficient Handoff in HAPS to Sustain QoS," presented at the 2012 IEEE Students' Conference on Electrical, Electronics and Computer Science(SCEECS-2014), 2014. https://doi.org/10.1109/SCEECS.2014.6804496 